Neuropathology and Prion Research, Ludwig-Maximilians-University, Munich, Germany

Glioblastoma multiforme (GBM) present with an abundant tumour neo-vasculature and a high degree of tumour cell infiltration into the parenchyma. In a serial implantation model recapitulating the angiogenic switch of human GBM we found that the expression levels of the proangiogenic receptor APLNR and its cognate ligand APLN strongly increase with the onset of tumour-vascularisation. Screening a set of murine and human GBM stem cell (GSC) cultures of different genetic subtypes we consistently detected APLN/APLNR expression. Knockdown of APLN (AKD) in these GSCs and subsequent orthotopic implantation in mice massively reduced the GBM vascularisation, as compared to tumours generated by control GSCs. Injection of AKD-GSCs into APLN-knockout mice further normalised the intratumoural vascular network to a level otherwise observed in the tumour-free brain. Infusion of the APLNRagonist apelin-13 restored angiogenesis in APLN-deficient tumours. In stereotactic biopsies of GBM patients we found an upregulation of APLNR in the tumour infiltrative zone (lacking aberrant vascularisation), which points towards an additional role for APLNR in tumor cells. In a series of cell culture and in vivo experiments we observed that autocrine APLN signalling promotes GSC-invasion. Importantly, application of an APLNR antagonist was able to block both tumour angiogenesis and GBM cell invasion in vivo. In summary, we show that APLN-APLNR signalling induces GBM neo-vascularization as well as GSC-invasion and that both pathological features are blunted by APLNR-blockade. We propose APLNR inhibition as a new strategy for combined anti-angiogenic and anti-invasive GBM treatment, which may provide therapeutic benefits for a broad range of GBM subsets.

\section{ANGI-03. THE MIGRATORY SWITCH - INVESTIGATING MESENCHYMAL-AMOEBOID TRANSITION (MAT) IN HIGH GRADE GLIOMAS}

Sophie Taylor ${ }^{1}$, Sabine Knipp ${ }^{2}$, Arndt Rohwedder ${ }^{2}$, Alistair Curd ${ }^{3}$, Nina Struve ${ }^{4}$, Michelle Peckham ${ }^{3}$, John Ladbury ${ }^{2}$, Susan C. Short ${ }^{5}$ and Anke Brüning-Richardson ${ }^{1}$; ${ }^{1}$ Leeds Institute of Cancer and Pathology Translational Neuro-oncology Group, Section of Oncology and Clinical Research Wellcome Trust Brenner Building, St. James's University Hospital, Leeds, United Kingdom, ${ }^{2}$ Cancer Research and Structural Molecular Biology - Ladbury Group School of Molecular and Cellular Biology, Faculty of Biological Sciences University of Leeds LIGHT Labs, Leeds, United Kingdom, ${ }^{3}$ The Astbury Centre for Structural Molecular Biology, School of Molecular and Cellular Biology, Faculty of Biological Sciences, University of Leeds, Astbury Building, Leeds, United Kingdom, ${ }^{4}$ Laboratory of Radiobiology \& Experimental Radiooncology, University Medical Center Hamburg Eppendorf, Hamburg, United Kingdom, ${ }^{5}$ Leeds Institute of Cancer and Pathology Translational Neuro-oncology Group, St. James's University Hospital, Leeds, United Kingdom

One of the most devastating hallmarks of cancer is cell migration/ invasion, a prerequisite for tumour metastasis. Targeting this cellular phenomenon offers an opportunity to improve the treatment of invasive and highly migratory tumours such as Glioblastoma multiforme, and to better understand the cellular mechanisms controlling cell migration. Previous work, using a range of migration assays and inhibitors targeting the actin polymerisation pathway, identified a compound, CCG-1423, that distinctively failed to halt migration in $3 \mathrm{D}$ invasion assays. We suggest that mesenchymal-amoeboid transition (MAT) is induced by CCG 1423 allowing the switch from one migratory modality to another. It is reported that CCG-1423 reduces the expression of CCN1; a key adhesion protein in mesenchymal migration, by blocking nuclear import of the transcriptional co-activator, MKL1. ELISA assays also confirm significantly reduced CCN1 levels following treatment with CCG-1423 in the established cell lines U251 and U87 compared to untreated controls. In addition, preliminary data using immunofluorescence and western blotting techniques, suggest an increase in MKL1 levels in the cytosol of CCG-1423 treated cells, which is consistent with the absence of MKL1 nuclear import, as previously reported. Phenotypically, CCG-1423 has been shown to induce MAT, visualised using Instant Structured Illuminated Microscopy (iSIM), a super-resolution microscope built and housed at Leeds University. With $150 \mathrm{~nm}$ resolution and rapid acquisition of Z-stacks, this technology has allowed us to observe in detail a change in morphology from elongated to rounded in treated versus untreated cells detached from the core of U251 spheroids embedded in collagen. Detailed analysis of the iSIM images will allow us to understand the effect of CCG-1423 on MAT and the signalling pathways involved. This will facilitate pharmacological intervention by the development of combination treatments to target both mesenchymal and amoeboid cell migration and to fully prevent glioma cell migration and invasion.
ANGI-04. HIGH RESOLUTION RNASEQ PROFILING OF GLIOBLASTOMA RESIDUAL INVASIVE CELLS ISOLATED BY A NOVEL 5ALA FLUORESCENCE BASED METHOD

Jonathan Rowlinson, Maria Estevez-Cebrero, Anbarasu Lourdusamy, Ruman Rahman and Stuart Smith; University of Nottingham, Nottingham, United Kingdom

INTRODUCTION: Glioblastoma (GBM) is a polyclonal tumor with molecular abnormalities varying temporo-spatially (intra-tumor heterogeneity), one mechanism of therapy resistance. Fluorescence guided neurosurgical resection is performed with prior administration of 5-aminolevulinic acid (5-ALA) leading to individually fluorescent tumor cells mixed within a background population of non-neoplastic cells in the invasive region beyond "pure" tumor. We have isolated this critical invasive population by 5ALAbased fluorescence activated cell sorting (FACS) to study invasive GBM without an overwhelming background "normal" signal. METHOD: We performed high-resolution RNAseq analysis on 45 specimens from 10 GBM patients including samples from tumor core, rim, invasive zone and GBM cells from within brain parenchyma by 5-ALA based FACS. Genome-wide gene expression analysis (Affymetrix) on 14 glioma samples from three different GBM patients was also undertaken, with validation by qPCR and immunohistochemistry. RESULTS: Fluorescent cancer cells accounted for an average of $1 \%$ of the samples subjected to FACS. High-resolution RNAseq profiles were generated for tumor regions and fluorescently isolated invasive cells. Statistical analysis by linear models identified 325 differentially expressed genes between FACS positive cells and other tumor regions (multiple test adjusted P-value $<0.05$ ), of these, 50 genes were upregulated in all comparisons. These transcriptomic changes orchestrate MAPK (DUSP1, DUSP2, DUSP10 and FOSB), chemokine signaling pathways (CXCL2, CXCL3, CCL20 and NFKB1) and negative regulation of cell proliferation (EREG and KLF6). In contrast, 29 downregulated genes in FACS positive cells were enriched with signal transduction (DDR2 and MTSS1L) and ECM-receptor interaction (COL4A1, COL4A2, and HSPG2). CONCLUSION: Residual GBM cells in the invasive zone are shown to exhibit activation of distinct molecular pathways and biological processes. These unique molecular features offer hope for developing more efficacious targeted therapies focusing on this population rather than the bulk tumor that has been the subject of most historical analyses.

ANGI-05. CYTOMEGALOVIRUS INFECTION STIMULATES TUMOR CELL-PERICYTE CROSSTALK TO FACILITATE ANGIOGENESIS AND TUMOR GROWTH IN GLIOBLASTOMA

Harald Krenzlin; Brigham and Women's, Boston, MA, USA

INTRODUCTION: Cytomegalovirus (CMV) has been linked to glioblastoma for over a decade. However there is a lack of understanding of the mechanisms by which CMV affects tumor growth in vivo. Here, we have identified novel regulatory mechanisms involving cross-talk between GBM cells and vascular pericytes in the context of CMV infection, increasing angiogenesis and tumour growth in a mouse syngeneic glioblastoma model. METHODS: This study investigates CMV expression in human glioblastoma samples, and establishes and characterizes a new CMV latent, orthotopic glioblastoma mouse model. RNAseq, and in vitro functional assays were used to investigate mechanisms. RESULTS: Immunostaining of patient samples identified viral antigens expressed in multiple cellular compartments including the novel observation of co-localization with pericytes. RNAseq of human brain vascular pericytes (HBVPs) and glioblastoma stem-like cells (GSCs) revealed upregulation of proangiogenic cytokines after CMV infection. Conditioned medium derived from CMV infected GSCs/HBVPs led to the establishment of larger $(160 \%, \mathrm{p}<0.0001)$ and more complex (number of junctions 9.5 (control) vs. $21(\mathrm{CMV}), \mathrm{p}<0.0001)$ tube formation in human brain microvascular endothelial cells (HBMEC) cultured on Matrigel in vitro. Also, PDGF-DD, secreted by GSCs after CMV infection, was identified as a driver of angiogenesis and HBVP migration in vitro. Orthotopic injection of Gl261fluc murine glioblastoma cells in C57BL/6 mice harboring latent CMV infection caused intratumoral virus reactivation and shortened survival rates (30d vs $43 \mathrm{~d}, \mathrm{p}=0.0004)$. Increased area $(\mathrm{p}=0.0004)$ and length $(\mathrm{p}=0.001)$ of tumor vessels was found in CMV latent mice. Furthermore, CMV infected tumours showed significantly higher numbers of infiltrating- and blood vessel-associated pericytes. CONCLUSION: In patient-derived tumor samples CMV partially co-localizes with perivascular pericytes. Tumor growth is faster in a murine glioblastoma model in the context of latent CMV infection, and this is in part driven by paracrine effects mediated by PDGF-DD which are associated with increased pericyte recruitment and angiogenesis.

ANGI-06. CHARACTERISATION OF INVASION OF HUMAN BRAIN TUMOUR INITIATING CELLS IN HUMAN GLIOBLASTOMA Verena Leidgens ${ }^{1}$, Maria Alieva ${ }^{2}$, Xin $\mathrm{Lu}^{3}$, Nina Patwary ${ }^{3}$, Eugen Kerkhoff ${ }^{4}$, Martin Proescholdt ${ }^{5}$, Arabel Vollmann-Zwerenz ${ }^{1}$, Jacco van Rheenen ${ }^{2}$, Christoph Klein ${ }^{3}$ and Peter Hau ${ }^{1} ;{ }^{1}$ Wilhelm 